\title{
Expanding the system definitions and configurations (taxonomy and data structure)
}

\author{
Magan Arthur \\ is the principal consultant at ACG - an independent consulting group for end-to-end planning and execution of \\ innovative enterprise content management projects.
}

Keywords: taxonomy, metadata, data structure, data presentation, metadata templates, polihierarchical structures

\begin{abstract}
This paper is part of a series of enterprise content management (ECM) best practices from ACG, an independent consulting group. The series provides practical tips and expert advice on topics covering planning, implementing, and improving enterprise content management systems and their components. This paper focuses on taxonomy and data structures. It is written from the point of view of the implementation team. It assumes you have some level of experience with the concept of metadata and taxonomies but it is not an academic study. This paper tries to be hands-on and intellectual only to the degree necessary to convey certain principles. It will provide links to resources which may also target more academic audiences. The complete ECM Best Practices Series from ACG is available at http:// www.arthurconsultinggroup.com.
\end{abstract}

\section{INTRODUCTION}

Taxonomy is the science of describing an object, in our case content or assets. In addition to describing the object, a taxonomy will also place it into a relationship with other content and group the content in logical collections or nodes of a hierarchy. ${ }^{1}$

Taxonomy is not a new term and library science is a good 2000 years old. The current renaissance is due to a growing understanding that file systems are not the right tool to manage and control access to the growing digital content repositories of companies, governments or any organization even of medium size. Stricter rules and regulations, specifically in the USA, require companies to improve the organization of their content, and taxonomy is a way to apply some very old and proven methods to a new form of managing content. While some old wisdom can help with the new challenges, there are various aspects of the new media which are not well covered in the age-old science. This paper will address both areas.

A taxonomy for a larger system will need to describe and group content from various sources in a logical but also useful way. This structure can become a complicated hierarchy with hundreds of nodes. If you plan for a larger system and you do not have a librarian on staff, you should seriously consider securing 
the services of a consultant. In addition, almost every industry conference (AIIM, Henry Stewart DAM Symposium and others) has dedicated seminar tracks for taxonomy.

This paper starts with a clarification of terms. This is necessary because there are not yet generally accepted standards or even guidelines for the terms used in describing taxonomies or data structures. (Interestingly, you will find that building a larger taxonomy is a lot about clarifying terms.)

I will then follow the order, also used by Ann Rockley in her outstanding book Managing Enterprise Content ${ }^{2}$, of distinguishing metadata between the categorization and individual data. First I will discuss classification or categorization of content and then provide ideas on how to build a metadata scheme for the individual assets or content (Ann Rockley refers to this as element metadata).

In the last part of this paper I will describe considerations in regard to expanding the system, which will touch on other data structures not commonly included in the taxonomy discussions. These data structures include user groups and roles, security, ingestion and download folder structures, as well as other searchable indexes.

\section{CLARIFICATION OF TERMS}

Before getting into more detail I would like to clarify a few terms.

Taxonomy is a system of describing an object also through its relationship to other objects. Usually these relationships are expressed in a hierarchy. Administrative data (use, usage rights, status etc.) are usually not considered in these definitions.
However, administrative data are very important for your system to function. There is a second meaning of the term taxonomy which is more broadly describing any data used to describe and classify content. It has become common to refer to any system used to find and describe digital content as taxonomy.

Metadata is a wider term which, for the purpose of this paper, shall include any data about an object both descriptive and administrative in nature (data about data).

Metadata structure is the system of metadata templates that will be used to classify, find and describe the objects of your system.

Collection will refer to any grouping of content which could be a folder, collection, or also a job or project.

Object will be any element that can be described with its own set of metadata: Individual files, collections, folders, jobs, projects, user groups, users, upload or staging folders and more. One way to think of an object is as a row in the database and metadata as columns.

Authoritative term is the term used to describe a node in the classification hierarchy. An authoritative term can have many synonyms or related terms but it is chosen to represent all these concepts as the most identifiable term in the classification.

Parent/child relationship expresses the hierarchical relationship in a classification. "Mammal" is the parent of "human," and "race" is the child of "human."

Ontology is a related term to taxonomy and usually tries to explain any object from its place in the hierarchy of other objects. I will try to avoid 
highly academic terms and instead use more descriptive language whenever possible.

\section{TAXONOMY OR} HIERARCHICAL STRUCTURE

\section{General considerations}

Before you start it should be said that common sense is a very important element in this exercise. The end result should be a structure that is easy to use by end-users, content contributors and administrators alike. A classification system for all content of a large organization is the best case scenario, but it might not be practical to maintain, as it requires ongoing maintenance from staff with specialized skill sets. If your key concern is a useful classification or search system for the daily tasks of the average person, your energy could be better spent on refining or "harmonizing" a number of smaller and more targeted structures managed by tools that are more departmental.

Another important clarification is that your "enterprise taxonomy" is not necessarily tied to a software product (existing or planned). It makes a lot of sense to start with a piece of paper. The following questions can be mapped in a spreadsheet or a simple table.

\section{Building the structure}

What constitutes content in your organization and where is it?

As you brainstorm this question you will almost naturally start building a classification in a hierarchical structure (taxonomy). This structure will likely resemble the structure of any content management solutions already in use and/or your existing file systems. In most companies, however, there is no agreed enterprise structure to the file systems or for different content management systems, digital or otherwise. Every department has different, sometimes poorly maintained, file folders.

Independent of any software solution you have or will employ to manage all or part of that content, creating a map of the content in your organization is a very valuable exercise. Figure 1 shows a sample structure.

Different users will make different logical associations and search for the same content in different ways. While for the sales team "images" might include anything from photos to logos and graphics, these are very separate categories for the professional designer. In the example in Figure 1, it would make just as much sense to build the hierarchy as shown in Figure 2.

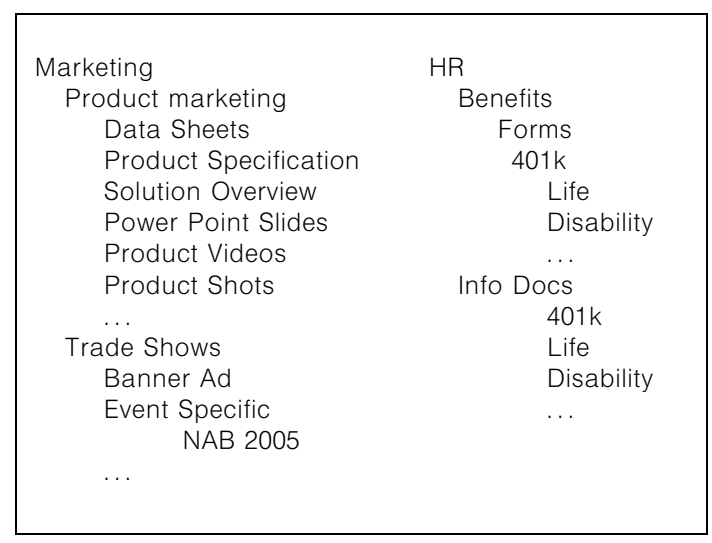

Figure 1: A sample structure

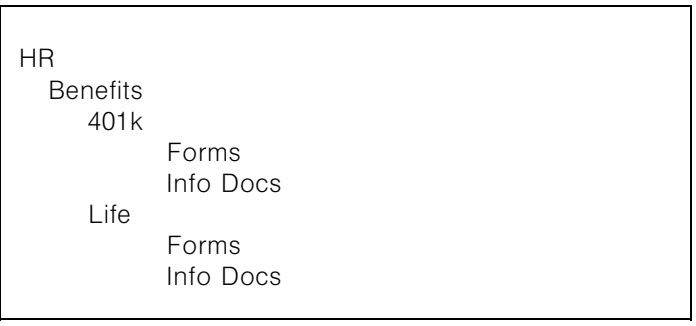

Figure 2: Alternative structure 
To begin with, these details are not that important. The first goal is simply to identify all the content that is of value for your organization. As with any larger project it is very important to have a general understanding of the scope and context. Only after that has been established will it make sense to decide in which area more detail and organization will most benefit the organization.

As you think more about your specific situation it will make sense to refine this general map. It is highly recommended that you involve the people who will ultimately use this system when you think about the following issues. This is not simply general good practice, but involving the users is essential to capturing both the formal as well as the informal relationships and flows of your content.

\section{Short-term versus long-term content}

As content will be ingested or cataloged into a more organized system it will have to follow specific rules. This can be work intensive and needs careful attention. It will probably not be necessary to catalog all content. Shortlived content with minimal potential for reuse is usually not valuable enough to be cataloged. For example, you might carefully catalog the high resolution version of your corporate identity images. But you will probably not need to catalog every low resolution rendition as any good digital asset management (DAM) system can easily create these on the fly.

\section{How deep do you need to go?}

In a library, every book gets a code that can be traced or browsed in the library's classification system. In other words, the

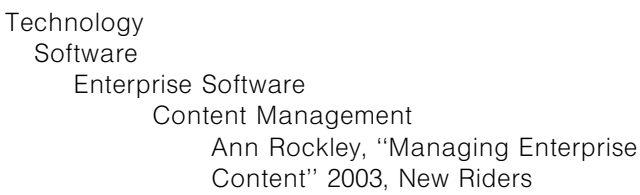

Figure 3: Library classification

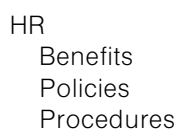

Figure 4: Simplified structure

last level of the hierarchy tree is a book (see Figure 3).

The ability of technology to display search results intuitively and to refine searches with specific metadata can make it slightly easier for a digital library. To use our example from Figure 2, the structure in Figure 4 might suffice to narrow the search to just a few items that can then be displayed as a list or any other useful representation (eg thumbnail) from which any user can easily pick the desired content or asset.

\section{Identify non-unique labels and build a} unique code

Another step to this exercise is to identify nodes in the hierarchy that have the same tile or name but that do not hold the same content. An example would be product specification. A marketing product spec and an engineering product spec will probably not contain the same information, but both can be found under the node "Product Specification." Most software systems that will manage a hierarchy will identify the elements of that hierarchy by a unique code. As we will discuss later, this has many advantages. It 
is therefore sensible for you to start thinking of a unique code scheme for your own system (eg

MAR_PROD_SPEC and

ENG_PROD_SPEC).

\section{Synonyms or "equivalence relationships"}

You should note all synonyms that are commonly used by your target users.

The best way to find out about this is to involve the users. Many systems fail because they are designed to fit the classification and are not built for and with the users.

In most cases you would want to define the following set of data:

- unique code

- authoritative term

- synonyms (including abbreviations and maybe even common misspellings).

Using the marketing product specification example, this is illustrated in Table 1.

\section{Parallel structures or "polyhierarchical taxonomies"}

Typically a classification hierarchy is represented in a hierarchical folder-like structure. However, it is important to note that unlike both the traditional library and the classic computer file system, the taxonomy is not a representation of the physical location of a file or asset. A relationship is built from the node of the hierarchy and the asset that is classified as part of that node. We need to think of this system more in terms of relational databases than bookshelves.

This difference provides possibilities that the analog library cannot provide.

For example, a file or asset can belong to more than one node in a hierarchy. The same book can simultaneously be on different shelves. As previously

mentioned, the way users will classify an object will vary depending on their specific perspective and need. Let's look at this example of an advertising agency.

The issue with these duplications is the maintenance of the hierarchy. If, in our example, a new version of a logo is created, it can automatically populate to all links as long as both hierarchies are managed by the same content management tool (Figure 5). However, if the studio creates a brand new logo for a client, it now also needs to be updated to the marketing "client logos" collection. While this can be defined as a process, it adds complexity. You will therefore have to weigh the advantage of cross-reference like the one above against the additional administrative overhead.

Table 1: Simplified database row for classification mode

\begin{tabular}{lll}
\hline Code & Authoritative Term & Synonyms \\
\hline MAR_PROD_SPEC & Marketing Product & Product Spec \\
& Specification & Product Specification \\
& & Data Sheet \\
& & Specs \\
& & Spec Sheet \\
& & etc \\
\hline
\end{tabular}




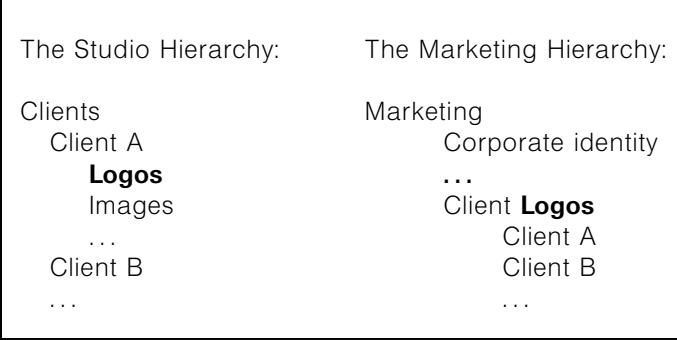

Figure 5: Duplication within classification

\section{Project-based classification}

It has become a generally accepted practice to have one authoritative classification structure that is carefully maintained by the administrator or librarian of the system. In addition to this structure, users might create specific subhierarchies that serve various purposes.

As long as new content is also cataloged into the authoritative classification hierarchy, anybody can find it.

This system makes sense also specifically for project- or job-based collections of assets. These project folders serve a different purpose than the overall classification hierarchy. They are often short-lived or created ad hoc. But they are very useful for that user or a small group of users to find content quickly in a specific context. Think of projects like "spring catalog" or also private folders for individual users that can help them group assets or content arbitrarily for their own needs (shopping cart, light boxes etc).

Let me stress again that you are not duplicating files or assets. You are simply referencing the asset in different organizational structures. Figure 6 simplifies the logical flow of this relationship between a file, the database record and the representation through organizational hierarchies or folders.

\section{Multiple systems}

In some cases you will not only duplicate the classification but you have separate tools you use to manage the

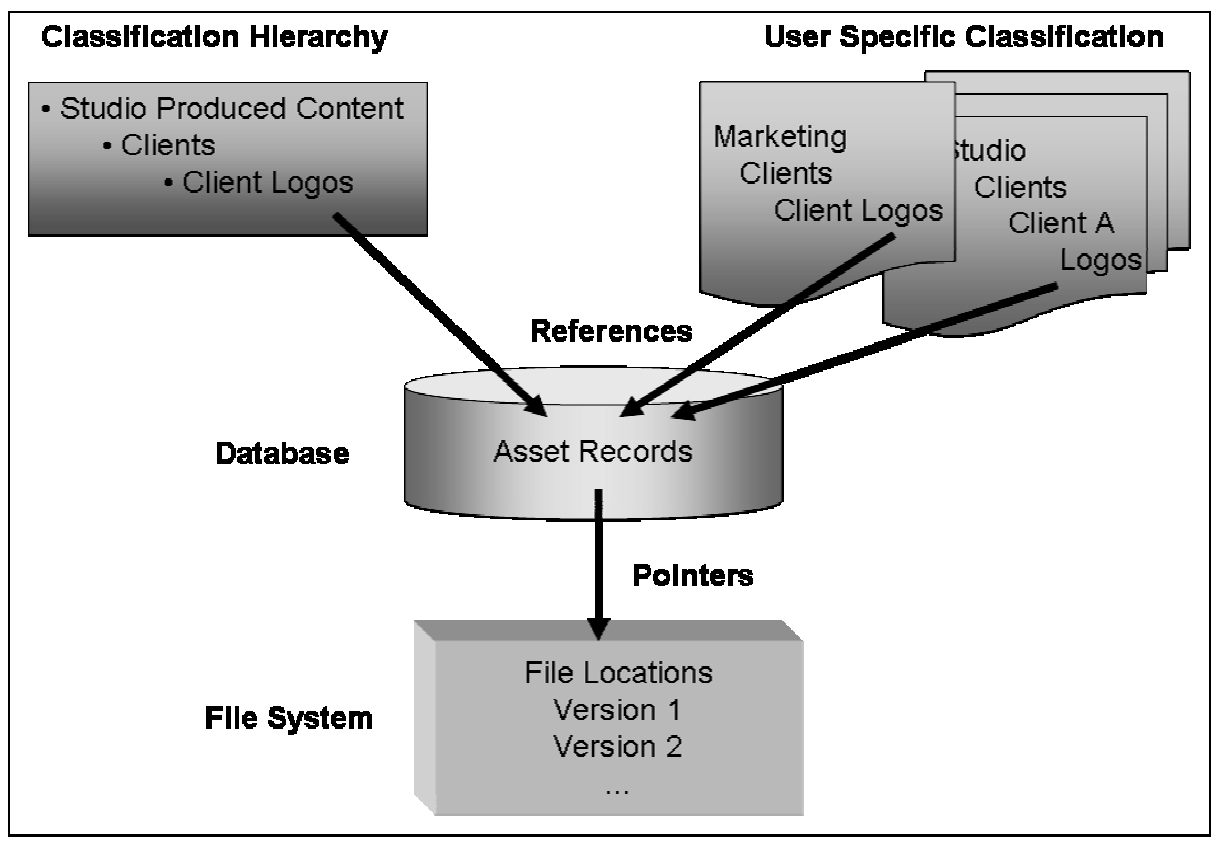

Figure 6: Data representation 
Table 2: Crosswalk

\begin{tabular}{llll}
\hline Description & Image Library & DAM Solution & Marketing Portal \\
\hline Name & File Name & File Name & File Name \\
& (Following our & (Following customer & (Following our \\
& naming convention) & naming convention) & naming convention) \\
Agency ID & N/A & File Name & N/A \\
& & (Following our & \\
Content Descriptors & Keywords & naming convention) & Keywords \\
\hline
\end{tabular}

content. An agency's studio might use a simple image library like Canto Cumulus or Extensis Portfolio for internal organization. The agency might now try to use more sophisticated DAM tools like ClearStory's ActiveMedia or Northplain's Telescope for client-specific projects and services.

Those and other more permanent duplications can be mapped with what is often called a crossover table or crosswalk.

\section{Crosswalk}

To manage any migration from one system to another you will need to make sure to map any relevant data as well. It will be of benefit to create a map similar to the one in Table 2.

\section{Taxonomy management tools}

Identifying relevant and interesting content and managing that content are not necessarily tasks of the same system. After reading the prior sections it should not be a surprise that there are software tools which are solely focused to manage taxonomies. These tools can read and feed the classification structures of various content management tools and some even allow you to link in with other publicly available resources. The communication is mostly accomplished via WebServices or Application Program Interfaces (APIs).
For example, consider a schoolbook publisher interested in the history of a specific people. His local taxonomy might have terms that are close to what he looks for, but it might not be a good fit for people's history. A domain connected to the local domain might be more on target (see Figure 7).

If you plan on this most sophisticated way for managing polyhierarchical taxonomies you should check out companies like:

- Synaptica: http://www.synaptica.com/

- Google (Enterprise Search Engines): http://www.google.com/enterprise/gsa/ index.html

- Verity: http://www.verity.com/

For the advanced user, Seth Eraley's paper "Managing Multiple Facets and Polyhierarchical Taxonomies" is great reading. ${ }^{3}$

Before we move on to the next topic I

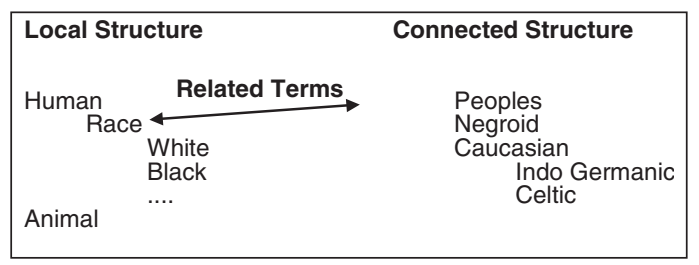

Figure 7: Related taxonomies 
would like to reiterate that the success of your project is measured by how much it will help users, contributors and administrators to manage content. This will depend very much on two elements:

1. How much were the users involved in the planning of the system?

2. The quality of the user interface design and usability of the tools and systems.

The first point is up to you. Below you will find a few points in regards to using and representing the taxonomy.

\section{Using or representing the structure}

There are many software tools available today that will manage classification hierarchies (taxonomies). Any document management (DM), larger web content management $(\mathrm{CM})$ or larger digital asset management (DAM) system will allow you to set up hierarchical structures to classify and manage content.

As discussed, there are also tools that are solely used to manage the classification scheme. They can be used in combination with the systems that manage the content repository. In either case the key is how the administrator can manage the hierarchy and how users can use it. More than anything, that is a question of representation.

\section{Representation}

As mentioned earlier, a classification hierarchy is typically represented in a hierarchical folder-like structure. It is, however, important to note that unlike the classic file system, this display is not a representation of the physical location of a file or asset.

In sophisticated systems, every node of the structure will become an object. As described earlier, an object is defined as something that can have metadata and therefore can be searched for. In other words, users can not only browse the hierarchy, but they can also search it.

We discussed the idea of unique codes and synonyms above. A good taxonomy tool will allow for searches such as "spec sheet." Following our example from above, this search will find the codes MAR PROD_SPEC and ENG_PROD_SPEC because it is a synonym of the main or authoritative term "product specification."

Table 3 looks at this search from the database perspective. The objects that the search for "spec sheet" would find can be expressed in simplified database rows.

Depending on the ability and flexibility of your tool, this can result in

Table 3: "Spec sheet" search result

\begin{tabular}{lllll}
\hline Unique ID & Code & Eng_Auth_Term & Eng_Syn & Parent ID \\
\hline Jh87837I & MAR_PROD_SPEC & Product & Product Spec & Jh673922 \\
& & Specification & $\begin{array}{l}\text { Marketing Spec } \\
\text { Data Sheet } \\
\text { (the ID } \\
\text { of Product } \\
\text { Specs }\end{array}$ & Marketing) \\
& & Spec Sheet etc & AK948322 \\
Jb958403 & ENG_PROD_SPEC & Product & Product Spec & (the ID of \\
& & Specification & Specs & Product \\
& & Spec Sheet & Documents) \\
& & Matrix etc & \\
\hline
\end{tabular}


Your search for "Spec Sheet" brought up the following choices. Check the term(s) that best match(es) your expected result and click submit to display the content associated with that term.

\begin{tabular}{|lll|}
\hline Root & Parent & Authoritative Term \\
Marketing & Product Marketing & Product Specification \\
Engineering & Product Documents & Product Specification \\
& & $\square$ \\
\hline
\end{tabular}

Figure 8: "Spec sheet" search result representation

any number of search result representations to your users. One common way is to present the root and the immediate parent of the term (Figure 8). In our example of a parallel structure the search for "logo" will provide the result shown in Figure 9.

In this case the user could find the same logos in different ways but this redundancy is not an issue as long as it is not confusing.

\section{Multi-language}

The concept of hierarchy objects that are identified by unique codes is also the key to multi-language display. Table 4 shows how a database might represent such an object.

This would allow a user to search for the term "spec sheet" in German (Technische Daten Beschreibung) either term would find the same content, because the content has a relationship to the classification term identified by the language neutral unique ID "Jh878371."

\section{Subject domains and synonyms}

Similar to the display elements above, a good interface of an advanced taxonomy

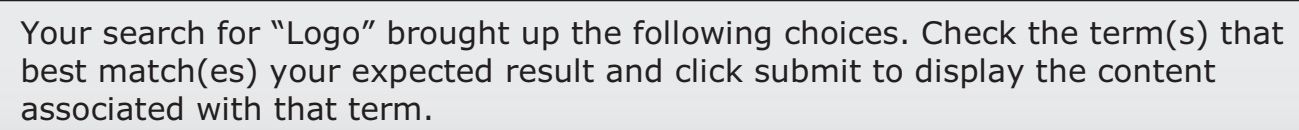

\begin{tabular}{|llll|}
\hline Root & Parent & Authoritative Term & \\
Marketing & Marketing & Client Logos & $\square$ \\
Clients & Client A & Logos & $\square$ \\
Clients & Client B & Logos & $\square$ \\
Clients & Client C & Logos & Submit \\
\hline
\end{tabular}

Figure 9: "Logo" search result representation 
Table 4: Multilingual database row

\begin{tabular}{llllll}
\hline Unique ID & Code & Eng_Auth_Term & Eng_Syn & Ger_Auth_term & Ger_Syn \\
\hline Jh87837I & MAR_PROD_SPEC & Product & Spec Sheet & Technische & Technische \\
& & Specification & & Produkt & Daten \\
& & & Broschüre & Beschreibung \\
\hline
\end{tabular}

management tool should provide options to explore a term in a hierarchy. In addition to parent and children, this interface should display synonyms, links to related terms of connected domains and possibly translations into foreign languages.

\section{Existing standards}

A number of existing taxonomy standards are listed below. These, as well as taxonomies created by companies in your sector (vertical taxonomies), can be a very good starting point. A good place to find other people in your industry sector who might have started content classification projects is at conferences ${ }^{4}$ or organizations. ${ }^{5}$

The following standards can offer more information:

- DCMI or Dublin Core: http:// dublincore.org - The best known taxonomy standard to date.

- IPTC: http://www.iptc.org/pages/ index.php - This is a standard used to include metadata in image files which is supported by many image software tools like Adobe Photoshop.

- EXIF: http://www.exif.org — This standard is used by many digital cameras to embed metadata into photography similar to ITPC.

- XMP: http://www.adobe.com/products/ xmp/main.html — An Adobe standard for embedding metadata into Adobe created files.
- MS property tags: http:// msdn.microsoft.com/library/ default.asp?url $=/$ library/en-us $/$ odc_wd2003_ta/html/ odc_wdcustprop.asp — The Microsoft standard to embed metadata into files created with MS Office applications.

- SMPTE 335M: http://www.smptera.org/mdd/rp210-2.pdf

- $M X F:$ http://www.mxfig.org/index.php

- $A A F:$ http://www.aafassociation.org

The above are metadata standards for television and broadcast.

- MPEG-7: http://www.chiariglione.org/ mpeg/standards/mpeg-7/mpeg-7.htm

- MPEG-21: http://www.chiariglione.org/ mpeg/standards/mpeg-21/mpeg-21.htm

Unlike the more known MPEG 1, 2, 3 and 4 these two are metadata standards for multimedia file description and exchange.

\section{Summary}

For a larger system, just defining the basic taxonomy can take weeks. There is no reason to wait until a decision for a product or vendor has been made. This classification will be a very useful tool for any vendor or consultant working with you.

At this point it will make sense to remember the opening paragraph. Use common sense when building your 
taxonomy. Many companies are realizing that content management at the enterprise level is a key strategy for long-term success. A simple inventory with a well-thought through structure is a good first step. However, classification is only one step of the process. In most cases users will not traverse long, potentially complex hierarchies to look for content. They will want to search by typing same basic values in a search page. This kind of search will require metadata.

\section{METADATA}

Simplified, you should be able to assign data to a file which will be used to describe and most importantly to find the file. These data are called metadata. There are different schools of thought about how to group these data. I find the following grouping most helpful.

Metadata can be

- Information about the file (objective) file size, type, color space, bit rate etc.

- Information about the content (subjective or user defined) - author, location, target audience, topic etc.

- Administrative information - approval status, storage path, lifecycle status, use etc.

- Information about the file's relationships - collections, parent documents, projects, jobs, inclusions etc.

\section{Building metadata templates}

You will find that different objects will need different data to be described and classified. A video's encoding type and compression are important, while MS Word documents will not need such information, although a value like "number of pages" might be helpful. I will provide a list of common data types and explanations below. If your system grows larger you might find that a hieratical composition of metadata templates for different categories of content makes much sense. In the example shown in Figure 10, an MS Word document would have the following data: classification ID, notes, keywords, file type, author, topic, page count, last printed.

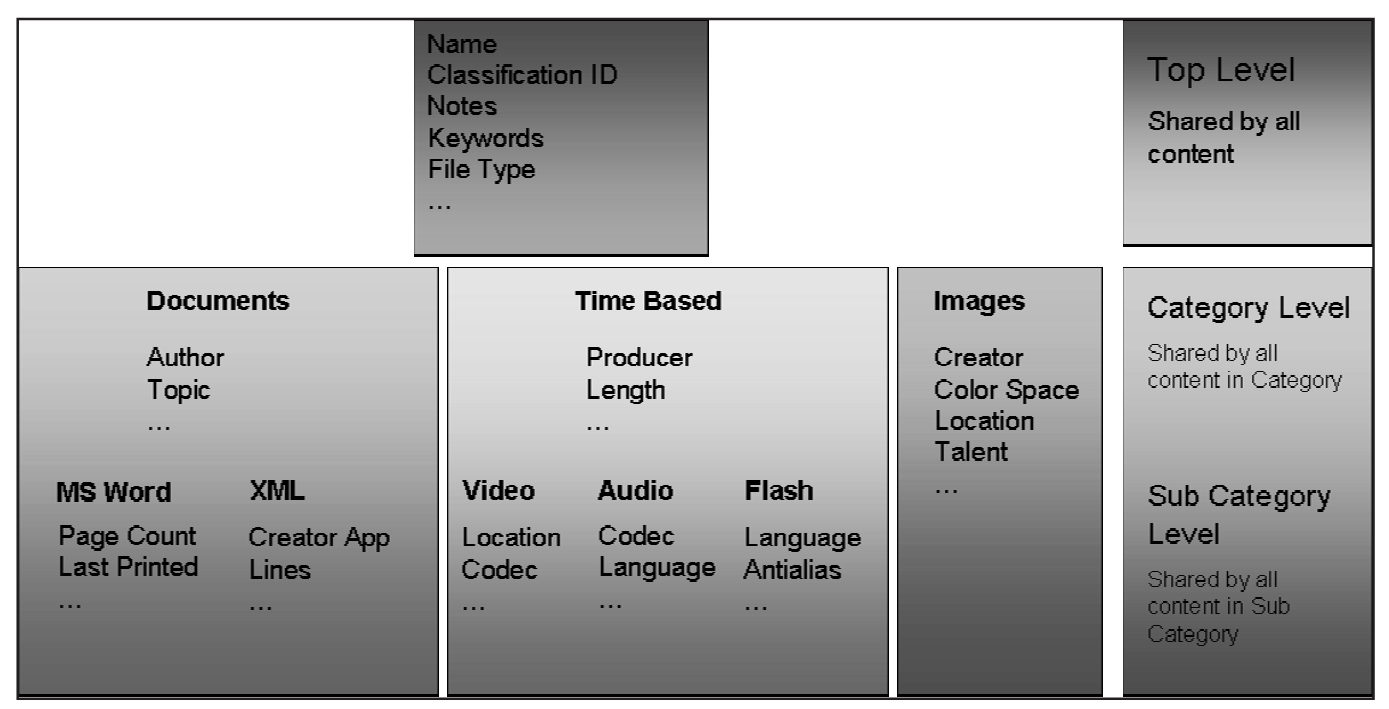

Figure 10: Hierarchical metadata templates 
Following are a number of questions that can help you building your metadata structure.

\section{What metadata do you need?}

The key question is which data do you need to assign to the different kinds of content or assets?

- Which data are needed for users and administrators to find an object?

Users are not only internal staff, they could be channel partners, consumers, investors, the press. It will often make sense to clarify which user group will use which data to find assets.

- Which data are needed to provide information about the object that users need but that are not used for searches?

This could be the file size or general notes.

- Which data might be needed in the future to find content or objects in an archive or in the later stages of its lifecycle vs. the earlier stages?

In some cases it might initially make sense to assign just a small set of data to an asset because time is of the essence; for example a fast turnaround of assets from a live event. Some of these assets might later become part of more permanent libraries and need additional metadata such as key words or usage descriptions.

It is important to realize that there is a big difference between the data that can be assigned vs. the data that are really needed by the users. There is a limit on how much data the average user and also the administrators can work with.

\section{How much data can you handle?}

- How much data can the users handle? In this regard it is of interest to note that few people have ever used the advanced search features of Google. Most users have limited time and even shorter patience. In order to become a useful tool, any system needs to be easy to use. Some complexity can be overcome by good search user interface (UI) design (this will be discussed later) but there is a limit to how much data a user can be expected to provide to find the content they are looking for. This also depends on the level of sophistication and training. Many systems fail because the search pages are designed with dozens of options and qualifiers. Most often less is more.

- How much data can the administrators handle?

There are options to support the administrator or librarian in keeping order with metadata, classification and cross references. These options are described in the next section. In many cases manual controls are necessary to keep the data "clean" and ensure searches reliably return all applicable objects. Thus, the administrator or librarian has an important job which will be detailed in the section about data integrity, below. As you design the system, you need to ensure that the administrative tasks are not becoming overwhelming or a bottleneck of system's efficiency. Of course, it is not solely the librarian that applies metadata. The processes of who assigns which data should be well defined and include the staff with the best context and motivation.

\section{How is the data assigned or applied?}

As defined above, there are different kinds of metadata. Below we go though the four categories and define who and how the data are applied. 
- Information about the file (objective) file size, type, color space, bit rate etc.

These data can in most cases be extracted automatically. This is good, but unfortunately these data are the least useful in identifying a specific file or asset. These data would be an "advanced" search option or simply information provided to users after they have found the content.

- Information about the content (subjective or user-defined) - author, location, target audience, topic.

Arguably these data are the most valuable for finding an asset by search terms. In most cases some of the subjective data are best provided by the content creator or someone very familiar with the context of the content. For example, caption text of a photo or keywords describing the content, are best defined by people closer to the context than the system administrator or general librarian. It could be the job of the latter to ensure data has been assigned in the right format but it is often hard for an "outsider" to ensure the data are correct.

Data about the content that has to do with business rules, such as owner or usage rights, can then often be defined by more administrative roles. The task of assigning the right data is therefore often a collaborative effort. In many cases administrative information can be used to manage this workflow.

- Administrative information - approval status, storage path, lifecycle status, use etc.

These data are either controlled by the system or by more administrative roles. A typical set-up would be to tag any newly ingested file automatically with a certain status, eg "new." This will then allow a dedicated librarian, information architect or other dedicated role to search for data with a "new" status and perform the necessary tasks and "flag" the asset with the applicable status for the next step, eg "reviewed."

If your organization is large and has a well-defined content management strategy with a defined enterprise taxonomy, you might have a dedicated person to control or manage this aspect. Figure 11 shows a sample content flow through the different classification and metadata assignment steps.

- Information about the file's relationships - collections, parent documents, projects, jobs, inclusions etc.

As shown in Figure 11, these data can be automatically assigned by the system, eg by using a dedicated upload folder that will assign pre-defined relationships to a project folder. Other examples include the upload of compound documents like Quark Xpress or InDesign. Those documents consist of many files that any good DAM system will link automatically in a parent/child relationship.

Throughout the content lifecycle, an asset or file might be assigned to other collections, folders, jobs and the like by users or administrators. This will most likely happen manually. We touched on this issue previously, in the section on project-based classification. Data integrity is vital: it is key to ensure all these data and the relationships will be useful and not confusing due to errors, omissions or misclassifications.

\section{How can you ensure data integrity?}

In the prior section we discussed options to automate the assignment of metadata. However, there is always a degree of human intervention necessary to fully classify and specifically describe visually 


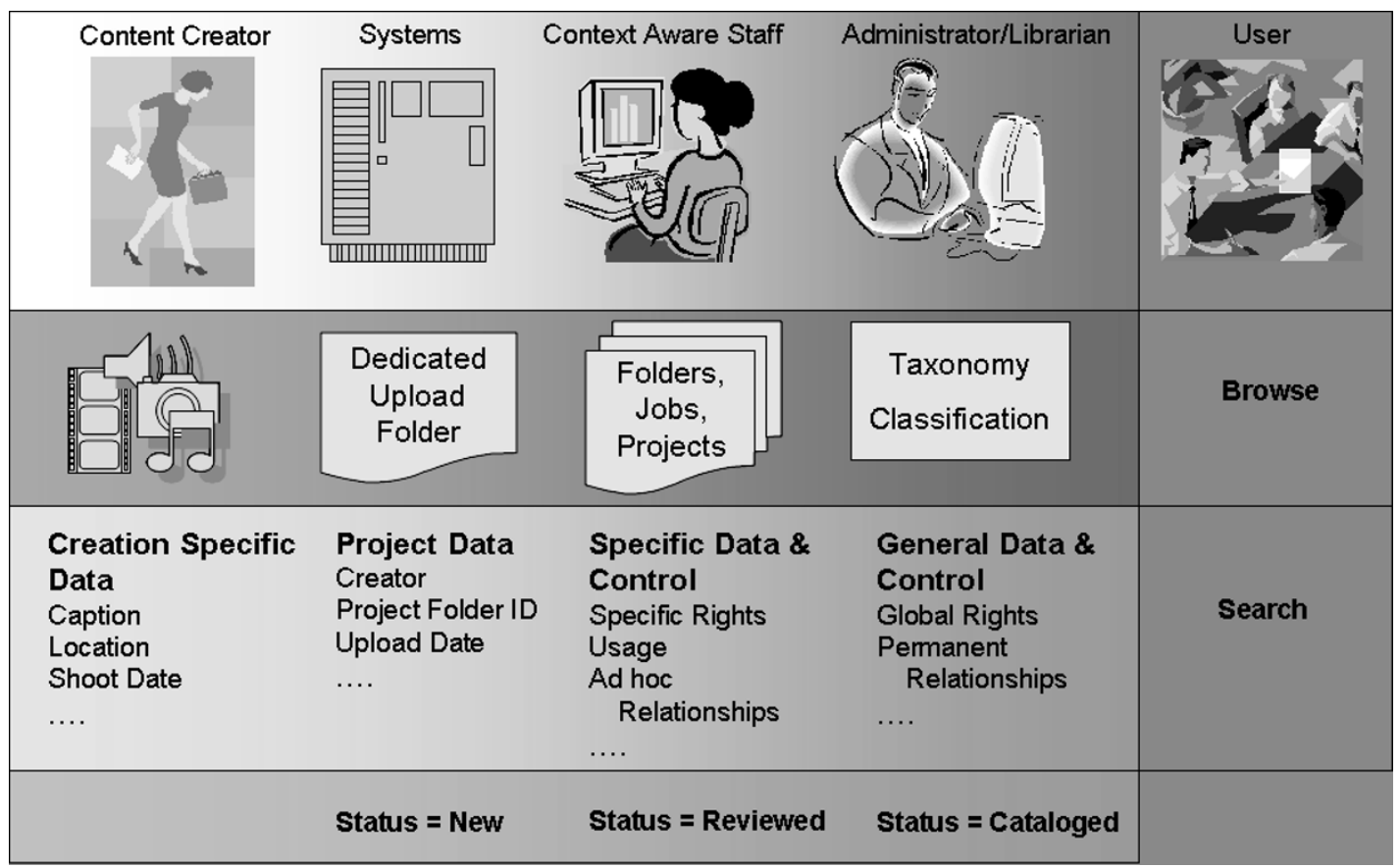

Figure 11: Classification and metadata assignment steps

rich content. The processes defined for the assignment of data are therefore very important. A basic rule is that there should be an incentive for the person assigning the data to do so with careful attention. Another aspect of data integrity is control. We will discuss that issue shortly.

\section{Content management requires dedication and skill}

No library would expect the average user to file books back into the shelf the margin of error would be too high. Following the example in Figure 11, the average creative worker can be expected to provide data vital for their work and drop the file into a dedicated "hot" folder, but one should not expect anything more.

Managing content should be defined and articulated as part of the job description of the staff responsible for setting up hot folders and assigning key data. We mentioned information architects (IAs - sometimes also called cybrarians) and libraries earlier in this paper. These jobs require dedication and skill. One common reason why systems do not archive with the expected effectiveness is because companies underestimate this aspect and leave the crucial management of metadata and relationships to unqualified and poorly trained staff. The level of data integrity and the effectiveness of the system is only as good as the dedication and skill of the staff managing it. Technology has only a limited role in this aspect. "Garbage in garbage out" has never been more applicable. If the cost of qualified staff is not part of the calculations for the projected return on investment, the calculations are flawed. 


\section{Controlled vocabulary}

One tool to ensure data integrity by administrators is that of controlled vocabulary. There are various ways to define a group of values for users to choose from, rather than just entering free text. These can be in the form of lists, hierarchies (again) or other controls. Both the value and the format of information can be controlled to some degree. While this can help to ensure data are assigned correctly, it will need additional thought and planning on the part of the system administrators. Controlled vocabulary options are highlighted in the list of common data types below.

\section{Control}

An administrator or librarian can take various measures to ensure that metadata have been assigned, and assigned correctly. We outlined two control steps in the process mapped in Figure 11. If the control is not part of the data assignment tasks at the time of cataloging, an administrator can simply search for an ingestion or catalog date range and inspect submitted material randomly or systematically (eg every 10th cataloged file).

Most systems will also allow searching for omissions. For example, a librarian could search for all content that does not have the classification Ibid.

Control can also be distributed to the areas of the organization that have a stake in certain aspects of the system. For example, in the logo scenario from earlier, the marketing department could and should have a dedicated person to check for new logos created by the studio to add to the marketing client logos folder.

While control and data integrity are very important for the usefulness and the adoption of the new tools and processes, an even more important element is the presentation of both search options and the resulting information and content.

\section{How is the data represented?}

This is a very important question. It is beyond the scope of this paper to discuss the UI recommendations and considerations in detail. However, it is fair to say that the UI is the ultimate milestone by which the usefulness of our system will be measured.

We have discussed various quite complex topics. In order to address the various user needs sufficiently, a system will need a way to build UIs (even a medium-sized system will have probably more than one) that are flexible and adoptable for change. It also needs to accommodate some freedom of creativity on the side of the designers. Most out-ofthe-box designs are targeted at one specific use case. If a tool does not provide some level of freedom in creating "customized" UIs, it is not a good tool for an enterprise application of any kind.

The figures used to depict hierarchies in this paper for example are all very dry and boring. There is no need to represent a hierarchy in such a way. Images or any more visual elements can become an intuitive and "fun" way to navigate. Fun is not necessarily a standard design guideline, but it should be. Flash and many other technologies allow users to move and adjust components on a website, thus creating a personalized experience that can actually be fun. This is a very important element of engaging the user and an invaluable contribution to the user acceptance and the success of the entire project.

In general we have three kinds of interfaces to think about: 
- search interfaces

- information display interfaces (search results)

- administrative or editing interfaces.

Below we will look at each in detail.

\section{Search interfaces}

A search actually starts before a user arrives at a search interface. It starts the moment a user clicks on a bookmark, enters a URL or chooses a specific application to look for something. It is therefore important to include these choices when analyzing the best interface approach for any system.

For example, when a user needs to pick a geographical location, this can be displayed in a long list or as an interactive map or animated globe that the user can click. The latter is sure to engage users much more intuitively. They might not even consider this "searching." After the user has arrived in California the search option could include entering a search term in a Google-like style or add some "advanced" data values (dates, file type etc).

Saved searches are also a great tool to make it easier for users to find specific assets. For example, a saved search can define even a very complex query and present it as a simple link on an intranet site. Consider the text "New logos and graphics for use in PowerPoint presentations" as a link on the marketing page. Such a link can hide the complexity of a specific query, eg $<$ Collection $=$ Marketing_Client Logos ORMarketing_New_Product_ Graphics \&Resolution $=$ low_res $\&$ Status $=$ Approved $>$.

Information display

How information is displayed is another important aspect of a good system. As with general interface design, the more targeted the information is for a specific user or use case, the better. When building display interfaces for users we need to know what they need to know. We need to understand both the kind of data that is required as well as the best format for that data. We will discuss later the various metadata types and formats that are commonly used. But the content itself can also have various renditions or proxies.

In the best case you will create a matrix of each user group and their requirements. Figure 12 is an example of a possible list for a user group.

\section{Administrative}

Administrators or librarians often have very different needs from the average user. At the same time, they should also spend more time in training and therefore should have a much better understanding of what the system can do. In some cases, an administrative interface is not a web browser but a thick client. The difference being that a

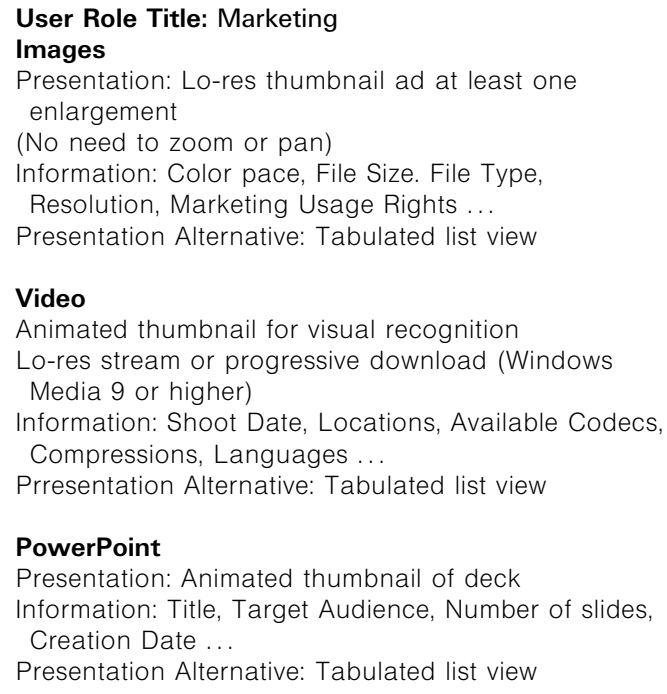

Presentation: Animated thumbnail of deck Information: Title, Target Audience, Number of slides, Creation Date.

Presentation Alternative: Tabulated list view

Figure 12: User role display definition 
thick client is an application installed on a local computer to allow more sophisticated actions, such as batch uploads and batch assignment of data or also editing of end-user interfaces.

A good system should allow administrators to build search pages on the fly by selecting from dozens of possible search values. These searches should be saved for later reuse or even published as links to end user. We discussed saved searches previously in this paper.

User and application security is another aspect of the administrator interface. Integration with exiting user administration tools such as iPlanet, Microsoft Active Directory ${ }^{6}$ or other LPAD based products is not the final answer to this issue. Many applicationspecific user administration tasks will have to be performed in any larger content management system. We will briefly mention how access control and security will also impact your taxonomy planning. But to complete this section on metadata we will list the common data types and the common objects that are defined by these data.

\section{Common data types}

\section{Descriptive}

- Pick one or pick many lists

These lists allow for controlled vocabulary. The biggest advantage of this data form is that it ensures the correct entry and spelling. It can be limiting if the list is not well-defined. In some cases systems can allow users to add to a list.

- Numeric (numbers, dates, currency)

- Alphanumeric (codes and unique IDs)

- Yes/no (Or boolean)

In this case it is important to verify that a system also can handle omissions. Administrators might need to search for assets where no value was assigned.

- Free text field

The most risk for user error and misspelling but common for notes, keywords, and caption.

- Controlled text fields

You can control the length or format of entered text. For example, you can check for the name@domainname.xxx email address format.

- Hierarchical (with or without inheritance)

Data types that are best expressed in a hierarchical manner are often overlapping into the classification area. However, it can make sense to allow users to pick one or multiple values from a hierarchical structure other than the main taxonomy. Inheritance means that a lower level value will automatically inherit the values of the nodes above. A human is always also a mammal. That inheritance flows down the tree, but inheritance can flow the other way round. Non-inheriting structures are more like computer file systems - you only get the value you pick.

A good example for hierarchical

\begin{tabular}{|lllll|}
\hline & Motive & Time of Year & Light & Atmosphere \\
Location & Huma & Winter & Sun & Fun \\
Urban & Animal & Spring & Shade & Romantic \\
Out Doors & Mammal & Summer & Half Shade & Love \\
Wild Nature & $\ldots$ & Autumn & $\ldots$ & $\ldots$ \\
$\ldots$ & & & $\ldots$ & $\ldots$ \\
\hline
\end{tabular}

Figure 13: Hierarchical subjects for images 
inheriting metadata is keywords for image libraries. When looking for an image hierarchies like these can be a great tool (Figure 13).

\section{Relationships}

In addition to descriptive and administrative metadata there are various relationships that can be important:

- Containers

- collections

- folders

- jobs

- projects

These containers for content or assets are objects that can be searched and organized just as individual assets can.

- Parent/child

An HTML page, a Quark XPress or Adobe InDesign document consists usually of a master template and various linked files.

- Lineage

This relationship usually tracks reuse as in a composite image (a new image created out of multiple photos) or between renditions that became individual assets. It is a mix of parent/ child and peer-to-peer.

- Versioning

Mostly a sequential linear relationship but this can become a complex relationship between different versions of a file and versions of the metadata. Versions can become hierarchical tree structures if different versions continue to evolve in parallel.

- Peer-to-peer

This relationship links assets without creating a new object like a folder or collection. An example is the domain relationship we discussed earlier in the paper (race is related to people).

\section{WHAT ARE THE OBJECTS YOU WILL NEED TO ASSIGN METADATA TO?}

As a final area of planning you will have to think about the different elements that need metadata. We defined those elements as objects. Most of this paper focused on the classification and description of content. However, other objects will need to be classified and grouped, and often they need metadata for searching and administrative tasks.

Here is a list of the most common elements that you will most likely have to include in your planning process:

- Files/content/ assets

$$
\text { - versions }
$$

- Containers

- collections

- folders

- jobs

- projects

- Users

- User groups

- Roles

- Upload or staging folders

- Nodes of the taxonomy tree (sometimes called subjects)

As previously discussed, a node of taxonomy can become an object which can be searched and which can have metadata such as synonyms and translations.

\section{TESTING}

In the planning for a larger system you should consider testing the system on a smaller scale. This is not always easy, because with limited content the users will often not find anything when searching for specific items. However, this defeats the purpose of providing a realistic testing environment. Effective pilot projects are therefore quite difficult 
to realize. Over the years, I have observed that a phased implementation approach is the best alternative. For example, start with building an image library or enable access for just one client through your services portal. The best first phases are those which are complete implementations with limited but well-defined scope. They can provide valuable feedback and build the competency of everyone involved over time.

\section{ACCESS CONTROL AND APPLICATION SECURITY}

In closing, I wish to mention briefly one area that is not usually considered as part of the taxonomy or metadata structure: access control and application security.

Even in a mid-size system of a few thousand assets, a user can be overwhelmed with the search options and the available information. Access control is not only a way to secure that assets are not accessed by unauthorized users. It is also a way to hide some complexity from the users. They will only see what makes sense to them. For example, a sales person will want the PDF of the marketing brochure. They do not need the native Quark XPress file of the same name and they surely don't need to see all the linked files that make up the end result. By assigning access right according to roles, the information can be filtered to the most applicable set of content.

\section{SUMMARY}

The implementation of content management systems is best accomplished in phases. This is not different for complex taxonomy structures. There is much value in building a larger enterprise taxonomy. As mentioned at the outset, this can be in the form of a spreadsheet or table. The implementation and with that the refinement of the details can be accomplished in phases. These phases should not be isolated projects. They should follow a larger strategy or vision but with each phase this strategy can and most likely will be adjusted to reflect "lessons learned.",

\section{References}

1. This website defines the term taxonomy in more detail: http://

www.mywiseowl.com/papers/

Taxonomy.

2. Rockley, A. (2003) Managing Enterprise Content, New Riders Press, Indianapolis, IN.

3. http://www.earley.com/Earley_Report/ ER_Managing_Multiple_Taxos.htm.

4. See eg http://www.gilbane.com/ or http://www.damusers.com.

5. See eg http://www.g-sam.org, http:// www.aiim.org and especially http:// www.cmpros.com.

6. http://www.microsoft.com/ windows2000/server/evaluation/features/ dirlist.asp.

7. Building a strategy for a unified content strategy is not easy but there are several experienced consultants in the field. You can find independent professional advice at http://www.g-sam.org, http:// www.aiim.org and especially http:// www.cmpros.com.

Note: All URLs last accessed 3 May 2005. 